\title{
5GC+: an Experimental Proof of a Programmable Mobile Core for 5G
}

\author{
Umberto Fattore, Fabio Giust, Marco Liebsch \\ NEC Laboratories Europe GmbH, Germany \\ E-mail: \{name.surname\}@neclab.eu
}

\begin{abstract}
The telecom industry is striving to evolve the current 4G system to the 5th generation of mobile networks, aiming at boosting data rates and performance for the traditional broadband services, as well as supporting a wide range of new use cases with different technical requirements. The first specifications of the 5G system are included in 3GPP's Release 15 and expected to be enhanced in the future Release 16. In order to cater for the diverse network requirements posed by the $5 \mathrm{G}$ use cases and in the view of instantiating customized networks that suit the vertical industry, a programmable and flexible data plane is envisioned as a key element of the future system architecture. In this context, this paper presents an advanced core network design underpinned by SDN principles, introducing enhancements to the current mobile networks' functional architecture and leveraging programmatic approaches for the agile provisioning and re-configuration of customized data planes. Our proposal is substantiated and validated by means of an initial experimental prototype, carrying out a functional proof of concepts including commercial 4G cellular base stations and devices.
\end{abstract}

\section{INTRODUCTION}

The incessant demand of Internet-based services has produced an unprecedented data traffic volume which keeps growing year after year. Such growth has been shown to be even more evident in mobile networks, registered to be 18fold in the past 5 years [1]. The trend is not supposed to slow down, given the huge penetration of $4 \mathrm{G}$ devices and the foreseen increasing number of $4 \mathrm{G}$ connections, and, in order to cope with the phenomenon, the telecom industry is constantly seeking to evolve their infrastructure, catering for higher data rates and enhanced services to their subscribers.

In line with such evolutionary path, in late 2015, the International Telecommunication Union (ITU) has finalized its vision of the $5 \mathrm{G}$ mobile broadband connected society, by setting the requirements and the supported uses cases for the future 2020 International Mobile Telecommunication (IMT) system $^{1}$. Along with the IMT-2020 road map set by ITU, the 3rd Generation Partnership Project (3GPP) has recently included 5G system specifications in its recent Release $15^{2}$.

The $5 \mathrm{G}$ system is expected to be very different from the previous generations. In fact, the proliferation of stationary and mobile devices with extremely diverse duty cycles, communication and mobility patterns, as well as energy constraints, is

\footnotetext{
${ }^{1}$ Refer to https://www.itu.int/en/ITU-R/study-groups/rsg5/rwp5d/imt-2020/ Pages/default.aspx

${ }^{2}$ refer to http://www.3gpp.org/NEWS-EVENTS/3GPP-NEWS/1937-5G_ DESCRIPTION
}

supposed to populate the new $5 \mathrm{G}$ ecosystem. This comes in response to a plethora of novel use cases from unconventional stakeholders and their associated business models, which pose a complex set of diverse network requirements, such as low latency communication, edge computing, as well as support of energy-constrained stationary and mobile devices [2].

Among the key features of the coming 5G systems, the industry demands programmability and flexibility: network operators seek mobile systems that allow faster service provisioning, easier maintenance, improved scalability, as well as the ability to customize networks and efficiently adapt to the mentioned vertical customers' requirements [2]. Software Defined Networking (SDN) and Network Function Virtualization (NFV) go hand in hand for the provisioning, maintenance and scale of infrastructure, network functions and services. For such characteristics, SDN/NFV have been employed by the research community to re-engineer the Evolved Packet Core (EPC), the current $4^{\text {th }}$ generation for mobile communications [3] and such effort has been a fundamental path to build the 5G specifications included in 3GPP's Release 15.

Taking into account the considerations above, in this paper we propose our advanced $5 \mathrm{G}$ Core $(5 \mathrm{GC}+)$, a mobile network core architecture that supports a programmable mobile core. Such an architecture leverages SDN to treat the data plane nodes distributed within the mobile network as policy enforcement points able to handle and steer user traffic based on policies such as for QoS, packet encapsulation, path switching, or charging. By doing so, the proposed data plane nodes incorporate the functions of today's mobile network gateways, introduce a much higher level of flexibility and are able to serve different radio access technologies (RATs). This work explores also the control plane counterpart needed to enable our novel data plane concepts. Our solution is validated by means of an experimental proof of concept, implementing the proposed core network design and employing commercial $4 \mathrm{G}$ base stations and devices.

In the rest of the paper, Section II gives an overview of mobile network architectures as specified by standard developing organizations (SDOs) as well as other related work. Section III presents our solution, describing the compatibility with $4 \mathrm{G}$ and $5 \mathrm{G}$ access technologies, the functional architecture and the operations. The description of the system's proof of concept and preliminary experimental results are found in Section IV, before concluding the paper in Section V. 


\section{ARCHITECTURES FOR THE MOBILE PACKET CORE}

The mobile networks' state of the art for large scale commercial deployments is still based on Long Term Evolution (LTE/LTE-A) and on the EPC [3], as 5G is still in a preliminary phase. In the EPC architecture, user data packets are transported by means of bearers, which consist on a chain of tunnels built with the GPRS Tunneling Protocol for User Plane (GTP-U) [4] between a Packet Data Network Gateway (PDN-GW, PGW) and a Serving Gateway (SGW) and between the SGW and the radio base station (eNB), which the User Equipment (UE) is currently connected to. Simply put, a bearer is a point to point connection between a UE and the PGW associated to the PDN (e.g., Internet) that the UE aims at connecting to. In order to gain access to additional PDNs, a new bearer for each PDN is set up, and other PGWs are associated to the user. A unique IP address is assigned to the UE for each bearer, and the corresponding PGW acts as the IP anchor for such address, thus creating a 1-to-1-to-1 mapping between the PDN to be accessed, the PGW for such PDN, and the UE's IP address to be used. One of the largest limitations of such architecture is that all user data packets between a UE and a PDN must traverse a single PGW, posing scalability problems and sub-optimal routing. Also, different PDNs require different PDN connections, thus exacerbating the complexity of the devices.

The 5G system architecture [5] introduces more flexibility by fully adopting the Control/User Plane Separation (CUPS) of the gateways: now the Session Management Function (SMF) in the control plane manages the User Plane Function (UPF) responsible for user packet forwarding. Specifically, session management in 5G allows two methods to bind a Packet Data Unit (PDU) session (i.e., the evolution of a PDN connection) to multiple PDU session anchors, i.e., UPFs interfacing the same or different data networks. The first method is through the SMF inserting one additional UPF in the data path, acting as uplink classifier able to steer user packets towards multiple PDU session anchors (UPFs). In the second method, a PDU session is associated to multiple IPv6 prefixes (i.e., multihomed), and each prefix is anchored at a different UPF. Also in this case, an UPF is inserted at a common point where all the data paths branch out, and such UPF is said to support branching point functionalities.

Leveraging multiple anchors based on the IPv6 prefixes associated to a connection is the baseline concept for the Distributed Mobility Management (DMM) solution in [6]. The DMM working group within the Internet Engineering Task Force (IETF) has pioneered the work towards evolving the architecture for mobile networks, as documented in [7], [8]. Besides the aforementioned group draft, [9] targets the CUPS problem space, by defining an abstraction of a forwarding device's configuration from the control plane's perspective, allowing for instance the latter to program the data plane through the usage of network controllers.

Beyond those from SDOs, many other ideas have been proposed, mainly targeting the $4 \mathrm{G}$ architecture but still mean- ingful towards the definition of 5G. The key point of such proposals is the implementation of the already mentioned CUPS and the introduction of SDN and NFV technologies, in order to obtain a flexible data plane programmed by the control plane. In this way, new scenarios are enabled in the core network (e.g. data packet routing optimization). The survey in [10] collects many of those proposals.

Nevertheless, softwarizing-enable technologies (i.e. SDN) typically lack of native support to GTP-U tunneling in the mobile core. Despite its recognized drawbacks, the GTP protocol is still considered in the latest 5G specifications [5] as the reference transport protocol. SBI (South Bound interface) protocols (e.g. OpenFlow) then need to be extended in order to be able to define forwarding rules for GTP-U encapsulated data packets. Despite some proposals go in this direction (such as [11], [12] and [13]), many other works argue for the advantages given by a GTP-less data plane. Advantages mainly are: (1) reducing the complexity and the overhead given by tunneling, (2) remove the central anchor node concept which is tightly related to GTP-U endpoints, (3) remove constrained options to optimize routing, etc. Some of these results are highlighted in [14]. In [15] a multi-dimensional aggregation solution for routing and classifying packets without GTP-U (and any other tunneling protocol) is also presented.

In our 5GC+ solution, we design a mobile core which includes all the enhancements introduced by $5 \mathrm{G}$, considering CUPS and implementing the so craved flexibility with the introduction of data plane programmability. On some aspects we even go beyond $5 \mathrm{G}$, enabling the use of a plain IP data plane (as proposed by [16]), offering deployment flexibility (e.g. no tunneling anchor) and routing efficiency. Finally, our system is designed in order to fully support 5G New Radio (NR) interfaces and at the same time keep strong backward compatibility with 4G Evolved UMTS Terrestrial Radio Access Network (E-UTRAN).

\section{A SOLUTION FOR A NOVEL DATA PLANE ARCHITECTURE}

\section{A. Achieving an efficient transition from $4 G$ to $5 G$ networks}

As with past generations of mobile networks, transitioning to the next technology requires a slow and costly network upgrade and equipment roll-out, especially targeting the Radio Access Network (RAN).

In light of the above, the 5G system specifications in release 15 include a mechanism to accelerate the penetration of $5 \mathrm{G}$, called Non-standalone deployment (NSA). With NSA, the user plane traffic of 5G-enabled mobile device is routed through the 5G New Radio base stations (called Next-Generation NodeB - gNB), offering enhanced data rates, whereas the control plane signaling transits via the legacy LTE. This way, the NR gNB can be deployed using the legacy EPC as backend infrastructure both in the control and in the data planes. On the contrary, with the 5G Standalone (SA) network and device standards and their evolution devised in 3GPP's Release 16, NR base stations as well as future enhancement to LTE base stations will be connected to the $5 \mathrm{G}$ core. 


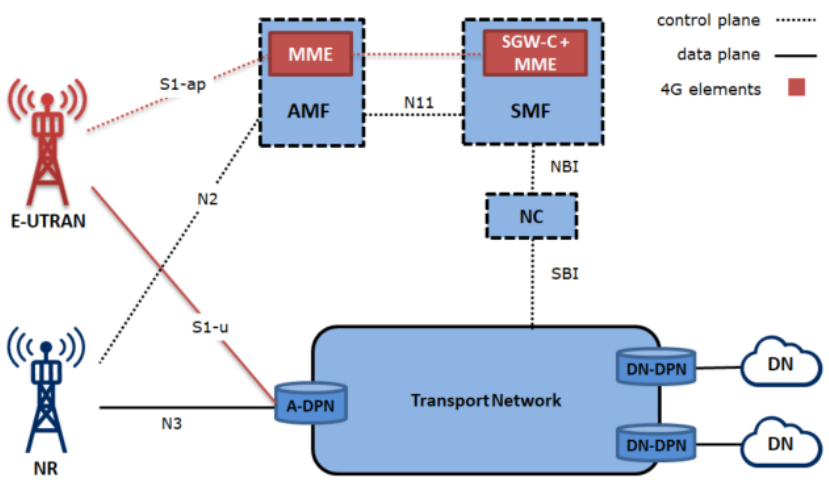

Fig. 1. 5GC+ supporting both $4 \mathrm{G}$ E-UTRAN and 5G New Radio

Despite the efforts made by the industry to enable a gradual switch over to 5G, current LTE and LTE-Advanced radios are envisioned to yet support a huge traffic growth, and it will take few years to see meaningful $5 \mathrm{G}$ shares of the total traffic volumes [1]. Nevertheless, the intrinsic EPC's characteristics make it poorly adequate to tackle future use cases, such as those involving edge computing. In order to cope with such scenario, we propose 5GC+, a unified architecture for the mobile core that connects the $4 \mathrm{G}$ and $5 \mathrm{G}$ RANs to a single and more flexible data plane. The purpose is to make some of the 5G technology enablers, like SDN and NFV paradigm also available to $4 \mathrm{G}$ access.

\section{B. Functional architecture of a mobile packet core to support dual RAN}

Fig. 1 illustrates the main components of 5GC+, the architecture proposed in this paper. The depicted architecture is designed aiming to support both $4 \mathrm{G}$ (E-UTRAN) and $5 \mathrm{G}$ (NR) access networks, using the standard interfaces from 3GPP [3], [5]. In the control plane, the architecture inherits two fundamental component from $5 \mathrm{G}$, which also map some 4G functions to support E-UTRAN as well. Those entities are:

- an Access Management Function (AMF), taking care of (1) registration management, (2) access authentication and authorization and (3) security context management, through the utilization of repositories storing user information. This element exchanges control plane messages with the NR through interface N2, and also implements part of functionalities of the 4G Mobility Management Entity (MME), such as terminating the S1-AP interface, whereas session management is devolved to the SMF;

- a Session Management Function (SMF), responsible mainly for (1) session establishment, modification, release and update, (2) IP address allocation for the mobile user and (3) data plane elements selection. It incorporates the MME functions related to session management as well as the SGW control plane (SGW-C).

Our proposal goes beyond standard 5G core architecture, by leveraging SDN in order to obtain flexible data path selection and data plane network programmability. This enables the SMF for many new additional data plane operations, such as
(1) selection of edge data plane anchors, (2) flexible relocations of the said edge anchors, (3) traffic steering between mobile user and services in order to select optimized routes.

An additional element between the control plane and the data plane is introduced in order to provide data plane abstraction: the Network Controller (NC). It receives traffic rules and policies from the SMF via a North Bound Interface (NBI), e.g., [9], and translates them to the data plane via the SBI, e.g. OpenFlow.

The aforementioned NC interacts with two different types of Data Plane Node (DPN):

- the Data Network DPN (DN-DPN), which is a forwarding device properly settled with traffic rules to access an external data network;

- the Anchor DPN (A-DPN), which is a forwarding device located at or in proximity of the access network, and equipped with some additional functions.

The A-DPN acts as an IP anchor for the the UE's traffic. The A-DPN is deployed at the edge of the network and so, in our 5GC+ solution, the IP anchor is no longer centralized as it was in $4 \mathrm{G}$ (i.e., the PGW). This allows for traffic routing optimization in the core network. In mobility scenarios, the control plane changes the A-DPN associated to the user traffic.

More, the A-DPN terminates the S1-U interface with E-UTRAN and the N3 interface with NR, so it must support GTP-U encapsulation protocol for data packet. Despite GTP$\mathrm{U}$ is still meant to be used in $5 \mathrm{G}$, we quote for a plain IP data plane in our solution (a possible data plane which achieves this is the Lean Packet System - LeaPS in [16]), in order to avoid the encapsulation effort and overhead given by using GTP or other encapsulation protocols. Therefore, the A-DPN also acts as an endpoint for GTP-U tunneling, allowing backward compatibility with the access network (both $4 \mathrm{G}$ and 5G). To establish the GTP-U tunnel towards the access network, the A-DPN needs some additional information to be forwarded by the control plane: therefore, the SMF needs to utilize a generic and extensible semantic and operation in order to support legacy GTP-U mechanism, at least towards GTP-only RAN elements.

\section{A closer look at the operational aspects}

In order to illustrate the operational aspects of the proposed 5GC+, Fig. 2 depicts the message sequence describing the UE attach procedure and the handover procedure (in which the ADPN is changed in order to maintain routing optimization). During the UE attach:

1) The RAN forwards an Attach Request to the AMF. This message contains UE subscription information and identifiers, as well as the service(s) requested by the user.

2) The AMF authenticates the mobile user through the AUSF (Authentication Server Function).

3) A Session Create Request is forwarded to the SMF, including all the information in previous message (1).

4) The SMF receives the request for a new session and allocates one or more IP address(es) for the UE session. 


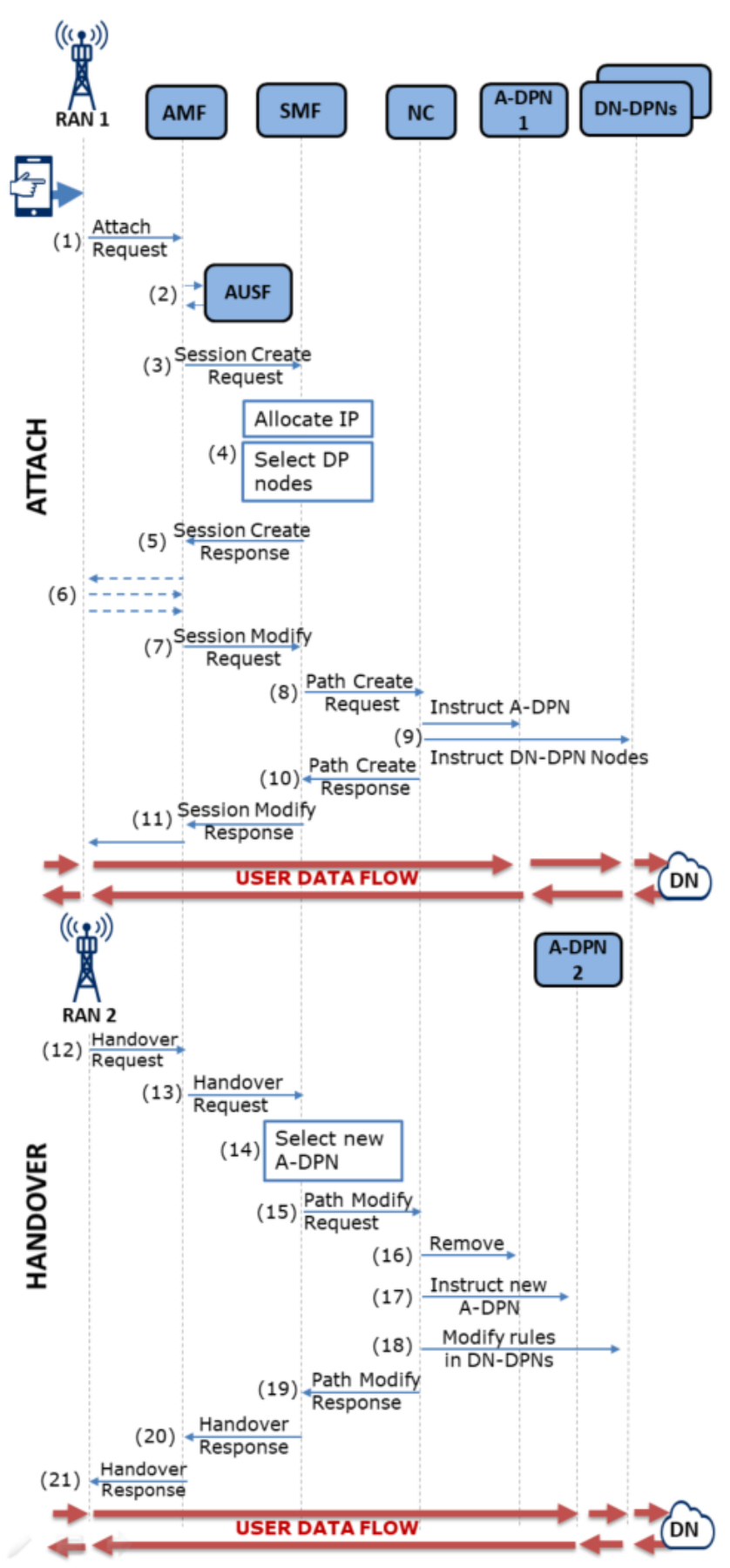

Fig. 2. Attach and handover procedures in 5GC+

More, the SMF selects the A-DPN node at the edge of the network based on its proximity to the device, and one or more DN-DPNs, based on the subscribed services or operator-specific policies.

5) A Session Create Response message is sent back to the AMF, containing the UE's IP address(es) and information (e.g. IP address, identifiers) to reach the A-DPN.

6) Further messages are exchanged between the AMF and the RAN to finalize the radio link setup. The latter is now able to reach the A-DPN, through the A-DPN's Tunnel
Endpoint Identifier (TEID) and IP address to establish the GTP-U tunnel. In this exchange, the RAN also sends back its own identifiers (TEID and IP address) in order to be reached by the core network.

7) A Session Modify Request is received by the SMF, which now holds all the needed information to build the complete data path.

8) With a Path Create Request message, the SMF delegates the NC to instruct the data plane nodes. Thus, this message contains indication of (a) the selected A-DPN, (b) the identifier (e.g. TEID tunnel) the A-DPN must use to be reachable from the access network, (c) the endpoint information to reach the access network and (d) the other DN-DPNs in the data paths.

9) The NC instructs both the A-DPN and the DN-DPNs with the appropriate traffic rules and policies. How such rules and policies look like depends on the type of transport implemented by the DPNs. For instance, in LeaPS [16], plain IP transport is used, with the traffic handling methods allowed by OpenFlow as SBI.

10) A Path Create Response message is sent back to the SMF as acknowledgment.

11) A Session Modify Response message is returned to the AMF. This message could be forwarded to the RAN as acknowledgment.

During a user session, an handover may occur e.g., due to user mobility. We consider the case of an handover requesting the selection of a different A-DPN and so the modification of the first segment of the data path. When the A-DPN changes, an important issue is to maintain IP session continuity for the UE, in order to avoid breaking ongoing device sessions. The following steps are performed:

12) A notification about the handover (triggered by the UE) is received from the access network.

13) An Handover Request is forwarded to the SMF, indicating the user session and the new access point.

14) The SMF selects a new A-DPN for the ongoing session. Again, this could be based on the proximity from the device or on other reasons (e.g., load balancing).

15) A Path Modify Request message is sent to the NC, indicating the new selected A-DPN, and (if present) other data path modification (e.g., QoS policy) requested for the UE traffic.

16) The NC deletes the rules regarding the specific UE from the previous anchor element A-DPN $N_{1}$.

17) The NC inserts the traffic rules in the new A-DPN (A$\mathrm{DPN}_{2}$ ) and the parameters to establish the GTP-U tunnel towards the new RAN element.

18) Finally, the NC modifies the traffic rules in the DNDPNs, in order to steer the user packets towards the new A-DPN. Once again, steering the traffic depends on the transport implemented by the DPNs. In case of LeaPS [16], Network Address Translation (NAT) is employed to re-direct packets through the new path, with no need to change the UE's IP address. 


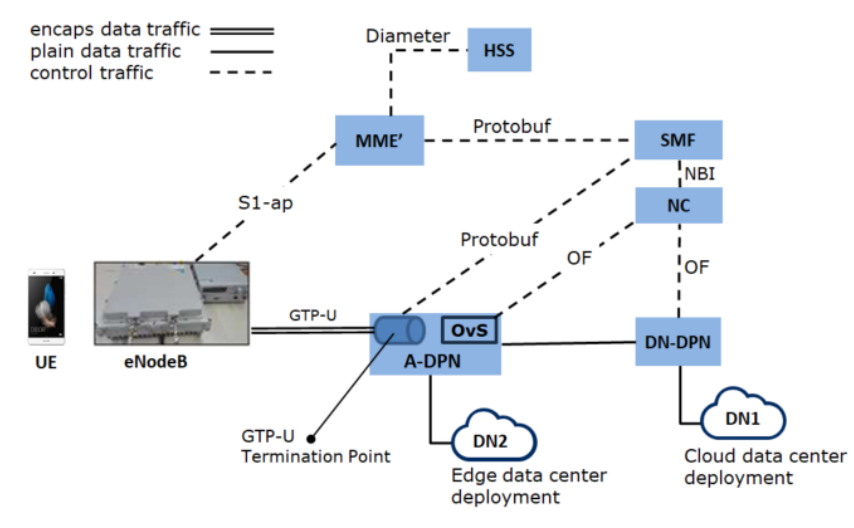

Fig. 3. Overview of the $5 \mathrm{GC}+$ testbed.

19) A Modify Path Response message is sent back to the SMF as an acknowledgement.

20) An Handover Response is returned to the AMF, containing the A-DPN endpoint information (i.e. GTP-U tunnel).

21) The information in (20) are forwarded to the RAN, in order to allow the data path establishment.

\section{EXPERIMENTAL VALIDATION}

\section{A. Testbed and software architecture}

The architecture presented in Section III has been implemented using OpenEPC ${ }^{3}$, which provides a 4G-compliant software platform with EPC components deployed in different virtual machines, managed by VMWare Workstation ${ }^{4}$. The original EPC components have been modified and extended in order to obtain the software architecture depicted in Fig. 3. In the radio part, a commercial eNodeB (NEC Orion eNodeB) and different commercial mobile devices have been used.

In the control plane, the testbed comprises the following:

- MME', which is obtained from a legacy MME by appending a GTP stub that terminates all the GTP-C signaling triggered by the MME. This way, the data path selection and session management functions are devolved to the SMF, trough a set of lightweight messages implemented with the Google Protocol Buffer ${ }^{5}$ and Protobuf-c ${ }^{6}$ tools, sent by the stub to the SMF. User authentication is still performed by the MME'.

- SMF, which is obtained extending the control plane functions of a legacy PGW, deprived of its data plane functionalities. The SMF receives from the MME' the request for a new UE connection, as well as the UE information for that connection. Its main roles are: (1) choosing an IP address for the UE, (2) selecting the data path for UE traffic, both in case of connection establishment and of A-DPN relocation, (3) instructing the NC to establish such data path through an NBI based on [9].

\footnotetext{
${ }^{3}$ https://www.openepc.com/

${ }^{4}$ https://www.vmware.com/products/workstation-pro.html

${ }^{5}$ https://developers.google.com/protocol-buffers/

${ }^{6}$ https://github.com/protobuf-c/protobuf-c
}

- NC, which is implemented as an OpenFlow v1.3 controller using the $\mathrm{Ryu}^{8}$ framework. It provides routing instructions to the DPNs, based on the indications received by the SMF.

- HSS, which is a legacy 4G Home Subscriber Server to perform user authentication.

In our implementation, we have built the transport network as a plain IP network, following the principles of LeaPS [16], and, additionally, we have integrated the cellular access. In order to do so, the A-DPN is obtained from the implementation of a PGW, from which the control plane has been removed and an Open vSwitch ${ }^{9}$ instance has been incorporated. DN-DPNs are plain OpenFlow switches.

Since OpenFlow v1.3 does not support GTP, we implemented a custom interface (using Protobuf-c) from the SMF to the A-DPN to convey: (1) the eNodeB's TEID and IP address to establish the downlink GTP-U tunnel towards the eNodeB; and (2) the A-DPN's TEID and IP address to create the uplink tunnel from the eNodeB.

Moreover, the A-DPN and the DN-DPNs are instructed by the NC (after indications from the SMF) with appropriate OpenFlow rules in order to accept the data packets associated to the UE's IP address.

In the testbed, two different data network (DN) deployments have been considered: a cloud data center deployment, in which the DN is deployed at a remote location outside of the mobile network domain, and an edge data center deployment, in which the DN is deployed at the network edge to enable edge computing scenarios.

\section{B. Preliminary EPC comparison results}

We validated our novel data plane concepts by verifying through experiments the correct support to the main UErelated procedures (attachment and detachment) and connectivity with the available commercial RAN, and showing the advantages of our $5 \mathrm{GC}+$ solution compared to legacy $4 \mathrm{G}$ when handling edge computing deployments.

In order to do so, we used 5GC+ and EPC to connect the UEs to the cloud data center and edge data center DNs, and we measured the round trip time (RTT) of the user data packets traveling from the UE to a web server in each of the two Data Networks (DNs). We added two artificial delays with the $t c$ (traffic controller) Linux tool ${ }^{10}$ : (1) $25 \mathrm{~ms}$ round trip delay on the link between the eNodeB and the A-DPN; and (2) $50 \mathrm{~ms}$ round trip delay between the A-DPN and the DN-DPN. In the EPC case, we used the OpenEPC's SGW and PGW, placed in conjunction with the A-DPN and the DN-DPN, respectively.

The left graph of Fig. 4 depicts the mean values of the measured delays, along with the 10th and the 90th percentile (represented with candlesticks in the graph). In the cloud data center deployment, both EPC and 5GC+ have similar

\footnotetext{
${ }^{7}$ https://www.opennetworking.org/wp-content/uploads/2014/10/openflowspec-v1.3.0.pdf

${ }^{8}$ https://osrg.github.io/ryu/

${ }^{9}$ https://www.openvswitch.org/

${ }^{10}$ from iproute 2 package (https://wiki.linuxfoundation.org/networking/iproute2)
} 

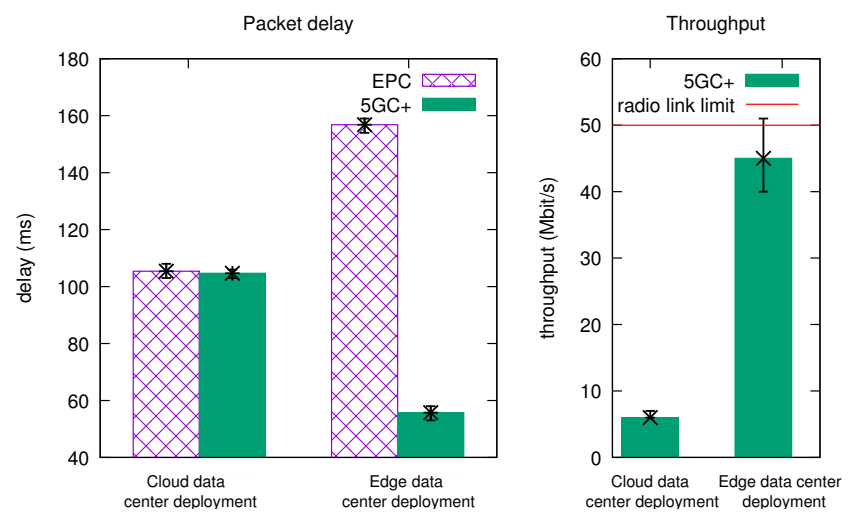

Fig. 4. Packet delay (on the left) and throughput (on the right) measurements.

delay values. This is due to the fact that in both cases data packets travel through the whole transport network to reach DN1. Nevertheless, 5GC+ outperforms EPC in the edge data center deployment, due to the lack of flexibility of the EPC architecture. Indeed, the PGW (centrally deployed in the core network and IP anchor for traffic in the EPC) forces data packets to be routed through it. This is avoided in the case of $5 \mathrm{GC}+$, where traffic is directly steered by the A-DNA to the near DN2, with evident benefits in terms of delay.

In order to further show the benefits of edge deployment facilitated by $5 \mathrm{GC}+$, we have measured the download throughput obtained when the UE connects to a web server hosted by the Amazon Web Services (AWS $)^{11}$ cloud and to a local web server sitting in proximity to the UPF. For this experiment, we have configure the LTE link to 50 Mbps in downlink, and, from the right graph of Fig. 4, we can see that with the edge deployment it is possible to obtain a throughput close to the radio link theoretical data rate. As for the first experiment, it is worth noting that $5 \mathrm{GC}+$ is able to efficiently support edge deployments, since is it natively designed for it, whereas legacy EPC architecture lacks support unless ad-hoc measures are implemented, like "bump-in-the-wire" traffic inspection as documented in [17].

\section{CONCLUSIONS AND FUTURE WORKS}

While the $5 \mathrm{G}$ definition is proceeding in the standardization track, the transition between $4 \mathrm{G}$ and $5 \mathrm{G}$ is at its very beginning. The NSA deployment only partially facilitates this transition. In this paper, we proposed 5GC+, a 5Gcompliant mobile core which fully supports this transition and which achieves next generation fundamental goals in terms of flexibility, as highlighted in some preliminary result. Beyond this, our 5GC+ enables a plain IP data plane network, allowing for integration with multiple data plane candidates for next generation core. The already mentioned LeaPS [16] is one of this candidates.

The integration of other data plane candidates besides LeaPS in our system is part of our future works. This requires changes in the control plane design as well as in the data

\footnotetext{
${ }^{11} \mathrm{https}: / /$ aws.amazon.com/
}

plane implementation. Nevertheless, a comparison between them would shed a light on potential benefits and drawbacks of each. More, other open issues have not been discussed in depth in this paper, which would enrich the functionalities of our proposed system. Among all, further investigations should address inter-RAT mobility and inter-RAT QoS support.

\section{ACKNOWLEDGMENT}

The research leading to these results has been partially supported by the H2020-MSCA-ITN-2016 framework under grant agreement number 722788 (SPOTLIGHT).

\section{REFERENCES}

[1] Cisco, "Cisco Visual Networking Index: Global Mobile Data Traffic Forecast Update," Cisco, White Paper, March 2017. [Online]. Available: https://www.cisco.com/c/en/us/solutions/collateral/service-provider/ visual-networking-index-vni/mobile-white-paper-c11-520862.html

[2] R. El Hattachi, J. e. Erfanian et al., "5G White Paper," NGMN, White Paper No. 1, February 2017. [Online]. Available: https:/www.ngmn.org/fileadmin/ngmn/content/downloads/ Technical/2015/NGMN_5G_White_Paper_V1_0.pdf

[3] 3GPP, "General Packet Radio Service (GPRS) enhancements for Evolved Universal Terrestrial Radio Access Network (E-UTRAN) access," 3rd Generation Partnership Project (3GPP), TS 23.401, May 2015.

[4] — , "General Packet Radio System (GPRS) Tunneling Protocol User Plane (GTPv1-U)," 3rd Generation Partnership Project (3GPP), TS 29.281, Mar 2017.

[5] _ "System Architecture for the 5G System; Stage 2," 3rd Generation Partnership Project (3GPP), TS 23.501, April 2017.

[6] C. J. Bernardos, A. De La Oliva, F. Giust, J. C. Zuniga, and A. Mourad, "Proxy Mobile IPv6 extensions for Distributed Mobility Management," IETF, I-D draft-ietf-dmm-pmipv6-dlif-00, April 2018.

[7] H. Chan, D. Liu, P. Seite, H. Yokota, and J. Korhonen, "Requirements for Distributed Mobility Management,' Internet Engineering Task Force (IETF), RFC 7333, August 2014

[8] D. Liu, J. C. Zuniga, P. Seite, H. Chan, and C. J. Bernardos, "Distributed Mobility Management: Current Practices and Gap Analysis," Internet Engineering Task Force (IETF), RFC 7429, January 2015.

[9] S. Matsushima, L. Bertz, M. Liebsch, S. Gundavelli, D. Moses, and C. Perkins, "Protocol for Forwarding Policy Configuration (FPC) in DMM," IETF, I-D draft-ietf-dmm-fpc-cpdp-10, March 2018.

[10] V.-G. Nguyen, A. Brunstrom, K.-J. Grinnemo, and J. Taheri, "Sdn/nfvbased mobile packet core network architectures: a survey," IEEE Communications Surveys \& Tutorials, vol. 19, no. 3, pp. 1567-1602, 2017.

[11] J. Kempf, B. Johansson, S. Pettersson, H. Lüning, and T. Nilsson, "Moving the mobile evolved packet core to the cloud," in Wireless and Mobile Computing, Networking and Communications (WiMob), 2012 IEEE 8th International Conference on. IEEE, 2012, pp. 784-791.

[12] W. Y. Pentikousis Kostas and H. Weihua, "Mobileflow: Toward softwaredefined mobile networks," IEEE Communications magazine, vol. 51, no. 7, pp. 44-53, 2013.

[13] M. R. Sama, L. M. Contreras, J. Kaippallimalil, I. Akiyoshi, H. Qian, and $\mathrm{H}$. Ni, "Software-defined control of the virtualized mobile packet core," IEEE Communications Magazine, vol. 53, no. 2, pp. 107-115, 2015.

[14] C. Sakshi and S. K. M., "Sdn based evolved packet core architecture for efficient user mobility support," in Network Softwarization (NetSoft), 2015 1st IEEE Conference on. IEEE, 2015, pp. 1-5.

[15] X. Jin, L. E. Li, L. Vanbever, and J. Rexford, "Softcell: Scalable and flexible cellular core network architecture," in Proceedings of the ninth ACM conference on Emerging networking experiments and technologies. ACM, 2013, pp. 163-174.

[16] F. Giust, A. Malik, and M. Liebsch, "Simplification as design principle for 5g: A native ip data plane for a lean packet system," in Personal, Indoor, and Mobile Radio Communications (PIMRC), 2017 IEEE 28th Annual International Symposium on. IEEE, 2017, pp. 1-7.

[17] F. Giust (ed.) et al., "MEC deployments in 4G and evolution towards 5G," ETSI, White Paper No. 24, February 2018. [Online]. Available: http://www.etsi.org/images/files/ETSIWhitePapers/ etsi_wp24_MEC_deployment_in_4G_5G_FINAL.pdf 